\title{
Optical characteristics of small surface targets, measured in the False Bay, South Africa; June 2007
}

\author{
Arie N. de Jong ${ }^{\mathrm{a}}$, Piet B.W. Schwering ${ }^{\mathrm{a}}$, Peter J. Fritz ${ }^{\mathrm{a}}$, Willem H. Gunter ${ }^{\mathrm{b}}$ \\ ${ }^{a}$ TNO Defence, Security and Safety, PO Box 96864, NL-2509 JG The Hague, Netherlands; \\ bInstitute for Maritime Technology (IMT), Martello Road, Simon's Town 7995, South Africa
}

\begin{abstract}
During the False Bay trial (June 2007), the performance of a set of three optical sensors was tested against several small surface targets in a coastal area: a hyperspectral camera, a camera with a rotating polarization filter and a high resolution camera. One objective was the validation of a contrast and clutter model for the visual spectral band in this type of scenarios. Another issue was to test the benefit of using a polarisation filter and a hyperspectral unit in front of standard TV cameras. Finally the loss in identification capability of a high resolution camera at long range due to atmospheric blur was investigated. Data were collected of targets in near-sun direction at ranges up to seven kilometers, in all cases for down looking angles (targets below the horizon). Environmental parameters such as solar irradiance and windspeed were measured as input for the contrast and clutter models. Spatial, spectral and temporal effects of the target contrast and of the background clutter behaviour in the visual spectral band were determined as function of range and compared with model predictions. Samples of data and predictions are presented in this paper. The spatial and temporal target characteristics are of key importance for the development of algorithms for target detection, classification and tracking. Finally, rules of thumb, based on the measurements and model predictions, on the detection and identification range performances of specific optical sensors against small surface targets in a maritime environment are presented.
\end{abstract}

Keywords: Target signatures, polarisation, hyperspectral imaging, target detection, optical blur, maritime scenarios

\section{INTRODUCTION}

Small surface target detection in coastal areas is of key importance in the war on drugs, encountering illegal immigration and piracy and rescue applications. In the last decade, a number of experiments has been set-up to collect data with electro-optical sensors in a variety of locations and conditions. TNO was involved in experiments in the Mediterranean Sea near Crete (1996, [1]) and Nettuno (1998, [2]), the Baltic Sea (1999, [3]), the Carribean (1999, [4]), the North Sea (2000, [5]), and Chesapeake Bay (2006, [6]). As a result a comprehensive data base has been obtained, which allows the validation of contrast and clutter models [7], the prediction of range performance for given sensor parameters [8] and the development of detection and tracking algorithms $[9,10,11]$. In these experiments the primary sensors were operating in the IR (midwave 3-5 $\mu \mathrm{m}$ and longwave 8-12 $\mu \mathrm{m}$ ), because of their day- and night capability. The target contrast in the IR is generally positive, due to cold sky reflectance of the sea surface, while the temperature of the target is close to the ambient air temperature. As a rule of thumb, the detection range corresponds to the range, at which the size of the target equals the projected pixel size at that range. The size of the target is generally enlarged due to the production of tumbling waves and wakes. Background clutter is dominated by the surface wave structure (affected by wind) and the sky radiance distribution.

Sensors, operating in the visual (and near-IR) spectral band, may provide substantial support to the IR sensors. Thanks to the small size of the detector elements (down to $5 \times 5 \mu \mathrm{m}$ ), TV-type camera's, provided with a $500 \mathrm{~mm}$ lens, have the potential (neglecting atmospheric blur) to give an angular resolution of $10 \mu \mathrm{rad}$, which corresponds to a spot size of $5 \mathrm{~cm}$ at $5 \mathrm{~km}$ distance. This type of resolution allows identification capability, not achievable with IR sensors. One drawback of the visual band (contrast loss by atmospheric scattering), is partially compensated by the lower cost of the sensor. In an early comparison [12] we recommended the use of a sensor package with near-IR and midwave IR sensors. In the False Bay trials, organised by IMT in June 2007 [13, 14, 15], we used a combined sensor package for the collection of signature data of a variety of small boats. While IMT was concentrating on midwave and longwave IR sensors, TNO was using a set of sensors in the visual band.

Infrared Imaging Systems: Design, Analysis, Modeling, and Testing XX, edited by Gerald C. Holst Proc. of SPIE Vol. 7300, 730003 - @ 2009 SPIE · CCC code: 0277-786X/09/\$18 · doi: 10.1117/12.816859 
The TNO sensor-set consisted of a hyperspectral camera, a standard camera with rotating polarisation filter and a camera with $400 \mathrm{~mm}$ lens for high resolution imagery. The objective of the first camera was to find out the general capabilities of the device and to determine the spectral emission from white caps and sunglints in particular. The second camera was used as a kind of simplified visual follow-up of an earlier experiment with an IR polarisation filter, described by Cremer [16]. The basic issue was here to find out what effects the use of the filter has on the target contrast and the background clutter, so what improvements in target detection performance we can obtain in certain conditions by using a rotating polarisation filter. The objective of the third camera was to see what spatial details of the targets can be discriminated in view of the blur measurements, simultaneously carried out over a fixed pathlength of $9.7 \mathrm{~km}$ (see [15]). In this paper a simple model is presented, describing the visual contrast of small surface targets at sea for given illumination conditions. In particular, some effort is spent on the theoretical aspects of using a polarisation filter. It is well known, that such a filter can reduce sky- and sunglints; however, a quantification of the benefit of the filter is hard to give.

\section{CONTRAST MODEL}

For consideration of the contrast of a target (or any object) at sea, the reflection properties of the water surface are of key importance. Just as a reminder of some basic optical laws (see for example [17]), the reflection properties at an interface are shown in Figure 1. Rays, incident on an interface between two media with refractive indices $\mathrm{n}=1$ (for air) and $\mathrm{n}$ (for water: $\mathrm{n}=1.33$ ) with an angle of incidence (with the normal) $\mathrm{i}$, are refracted with an angle of refraction i'. The reflection coefficient is dependent on the direction of polarisation. For light with a direction of polarisation normal to the plane of incidence (the plane through the incident ray and the normal), the reflection coefficient is $r_{n}$; for light, polarised parallel to the plane of incidence, the reflection coefficient is $r_{p}$. The mean value of $r_{n}$ and $r_{p}: r=\left(r_{n}+r_{p}\right) / 2$ as function of $i$, can reasonably well be approximated by the second order polynome: $r=7.146 * i^{2}-17.636 * i+11.037$ for values of $i$ between $70^{\circ}$ and $90^{\circ}$. For smaller angles of incidence, the reflection from the water surface will be neglected. Figure 1 also shows the polarisation index PI, here defined as $2 *\left(r_{n}-r_{p}\right) /\left(r_{n}+r_{p}\right)$, being a measure for the degree of polarisation.

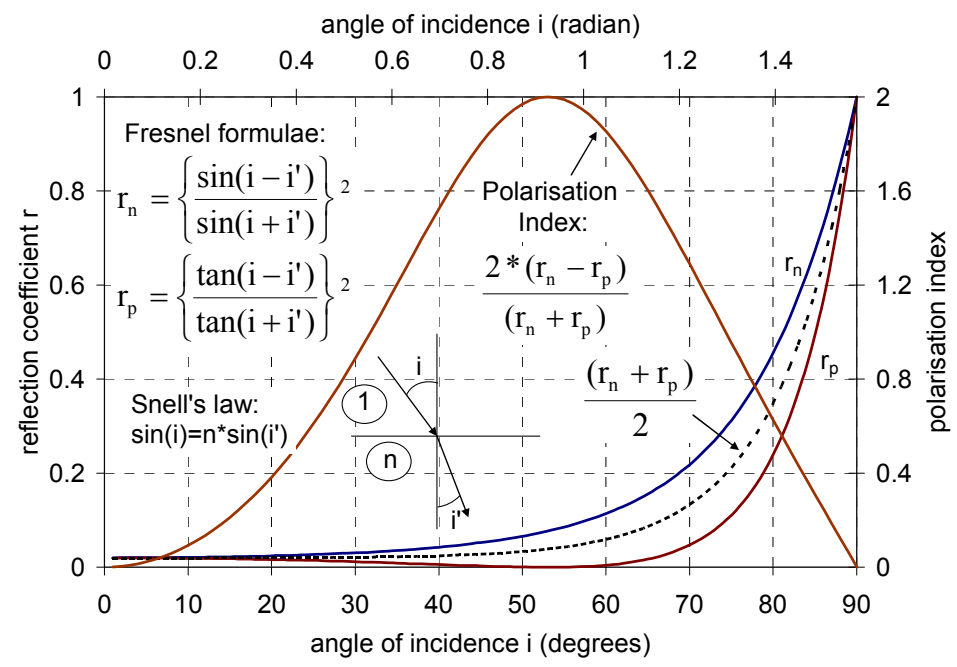

Figure 1. Illustration of basic reflection effects on a water surface
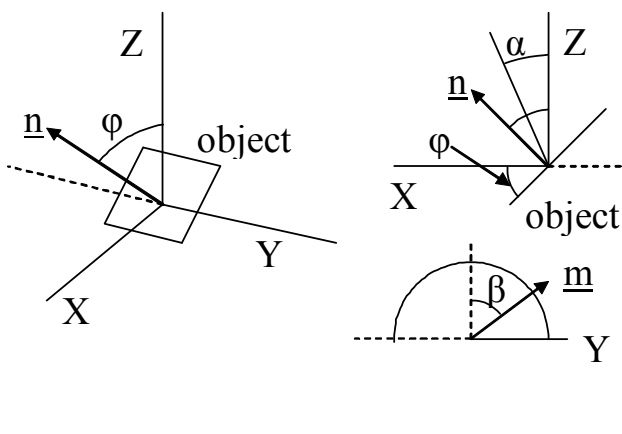

Figure 2. Geometry for deriving irradiance level at object by sky and sea irradiation

If we consider an object at sea, of which the normal $\underline{n}$ makes an angle $\varphi$ with the Z-axis (Figure 2), while the X-Y plane represents the water surface, the irradiance level of this object can be calculated directly under a couple of assumptions. The sky radiance $\mathrm{R}_{\text {sky }}$ will be considered to be homogeneous over the hemisphere. Radiance from the sky, reflected by the sea: $\mathrm{R}_{\text {sea }}$, will be calculated by using the approximation formula for $\mathrm{r}$. Solar irradiation can be estimated from the solar zenith angle, determining the air mass [18], and the angle between the sun-direction and the normal $\underline{n}$. At first we consider a flat sea surface. In the case of a non-flat sea surface, the reflection from the sea will decrease. Reflections of directed sunlight via the sea surface will be neglected. The surface of the object is considered as being a perfectly diffuse reflector,so its radiance level $\mathrm{R}_{\mathrm{t}}\left(\mathrm{W} / \mathrm{m}^{2} \mathrm{sr}\right)$ is the total irradiance level $\mathrm{W}_{\mathrm{t}}$ times $\rho_{\mathrm{o}} / \pi$, $\rho_{\mathrm{o}}$ being the reflection coefficient. 
Under these assumptions we can calculate the total irradiance level $\mathrm{W}_{\mathrm{t}}$ of the object from the three different irradiance contributions: $\mathrm{W}_{\text {sky }}, \mathrm{W}_{\text {sea }}$ and $\mathrm{W}_{\text {sun }}$, where $\mathrm{W}_{\text {sun }}=\mathrm{W}_{\text {sun, }}{ }^{*} \cos (\psi) . \mathrm{W}_{\text {sun, }}$ is the solar irradiance normal to the rays (as given in [18]), and $\psi$ is the angle between the sun direction and $\underline{\mathrm{n}}$. For angles $\psi>90^{\circ}, \mathrm{W}_{\text {sun }}$ is taken zero. For example, for an air mass of 1 , the solar irradiance level is found to be about $400 \mathrm{~W} / \mathrm{m}^{2}$ for the visual spectral band between 400 and $700 \mathrm{~nm}$. If one takes the output of a solarimeter (irradiance at a horizontal level), a correction has to be made from the solarimeter spectral band to the visual band including a conversion towards the irradiance level normal to the rays (divide by the cosine of the zenith angle). Thus we find: $\mathrm{R}_{\mathrm{t}}=\left(\mathrm{W}_{\text {sun }}+\mathrm{W}_{\text {sky }}+\mathrm{W}_{\text {sea }}\right) * \rho_{\mathrm{o}} / \pi$. To find $\mathrm{W}_{\text {sky }}$, we have to integrate over the angles $\beta$ and $\alpha$, shown in Figure 2, resulting in the following relationship of $\mathrm{W}_{\text {sky }}$ with $\varphi$ :

$$
\mathrm{W}_{\text {sky }}=\mathrm{R}_{\text {sky }} * \int \cos ^{2}(\beta) \mathrm{d} \beta \int \cos (\alpha-\varphi) \mathrm{d} \alpha=\mathrm{R}_{\text {sky }} *(\pi / 2) * \int \cos (\alpha-\varphi) \mathrm{d} \alpha=\mathrm{R}_{\text {sky }} *(\pi / 2) *\{1+\sin (\pi / 2-\varphi)\} \quad\left(\mathrm{W} / \mathrm{m}^{2}\right)(1)
$$

where the integral over $\beta$ runs from $-\pi / 2$ to $\pi / 2$, and over $\alpha$ runs from $-\pi / 2+\varphi$ to $\pi / 2$. In a similar way we can find $\mathrm{W}_{\text {sea }}$ by taking the relation between $\mathrm{r}$ and $\mathrm{i}$, mentioned before and replacing $\mathrm{i}$ by $(\alpha-\pi / 2)$ :

$$
\mathrm{W}_{\text {sea }}=\mathrm{R}_{\text {sky }} *(\pi / 2) * \int\left\{7.146^{*}(\alpha-\pi / 2)^{2}-17.636^{*}(\alpha-\pi / 2)+11.037\right\} \cos (\alpha-\varphi) \mathrm{d} \alpha \quad\left(\mathrm{W} / \mathrm{m}^{2}\right)(2)
$$

in which the integral over $\alpha$ runs from $\pi / 2$ to $\pi / 2+\pi / 9$. After integration, (2) becomes:

$$
\mathrm{W}_{\text {sea }}=\mathrm{R}_{\text {sky }} *(\pi / 2) *\{-0.175 \sin (\pi / 9-\varphi)-14.135 * \cos (\pi / 9-\varphi)+13.326 * \cos (\varphi)+4.814 * \sin (\varphi)
$$

Both $\mathrm{W}_{\text {sky }}$ and $\mathrm{W}_{\text {sea }}$ are plotted as function of $\varphi$ in Figure 3 for angles between $\pi / 4$ and $3 \pi / 4$ radians. It is found that for a vertically oriented object, the contribution to the irradiance from the sky is about 7 times bigger than the contribution to the irradiance via the sea reflection. We are now able to calculate the contrast $C$ of the object radiance $R_{t}$ with that of the sea background $R_{b}$, defined as $C=\left(R_{t}-R_{b}\right) / R_{b}=-1+R_{t} / R_{b}$. It is noted that we have to take a different value for the radiance of the background than in the case of the object irradiance, because we have to deal here with the look-down angle of the observer, which may be less than a few degrees below the horizon. The reflection coefficient $\rho_{\mathrm{s}}$ of the sea is high in this case, as shown in Figure 1. For the contrast $\mathrm{C}$ we thus find:

$$
\mathrm{C}=-1+\left\{\rho_{\mathrm{o}} *\left(\mathrm{~W}_{\text {sun,i }} * \cos (\psi)+\mathrm{W}_{\text {sky }}+\mathrm{W}_{\text {sea }}\right)\right\} /\left(\pi * \mathrm{R}_{\text {sky }} * \rho_{\mathrm{s}}\right)
$$

For $\varphi=\pi / 2$ (vertical plate) this relation reduces to: $\mathrm{C}=-1+\left(\rho_{\mathrm{o}} / \rho_{\mathrm{s}}\right) *\left[0.572+\left\{\mathrm{W}_{\mathrm{sun}, i} * \cos (\psi) /\left(\pi^{*} \mathrm{R}_{\mathrm{sky}}\right)\right\}\right]$. If the object is not directly irradiated by sunlight, this relationship reduces to: $C=-1+0.572 *\left(\rho_{\mathrm{o}} / \rho_{\mathrm{s}}\right)$. This implies, that the contrast $\mathrm{C}$ is only positive if $\left(\rho_{\mathrm{o}} / \rho_{\mathrm{s}}\right)>1.75$. From Figure 1 we find that for the grazing angles, that are considered here (angles of incidence greater than $\left.80^{\circ}\right), \rho_{\mathrm{s}}$ is greater than 0.4 , so only if $\rho_{\mathrm{o}}$ exceeds the value 0.7 , the contrast is positive. In Figure $4, \mathrm{C}$ is shown as function of $\rho_{\mathrm{o}}$ for various $\mathrm{W}_{\text {sun, }} / \mathrm{R}_{\text {sky }}$ values, $\varphi=\pi / 2, \rho_{\mathrm{s}}=0.6$ and $\psi=\pi / 4$. It is found, that even for moderate solar irradiation, $C$ tends to be negative if $\rho_{\mathrm{o}}$ is smaller than 0.4.

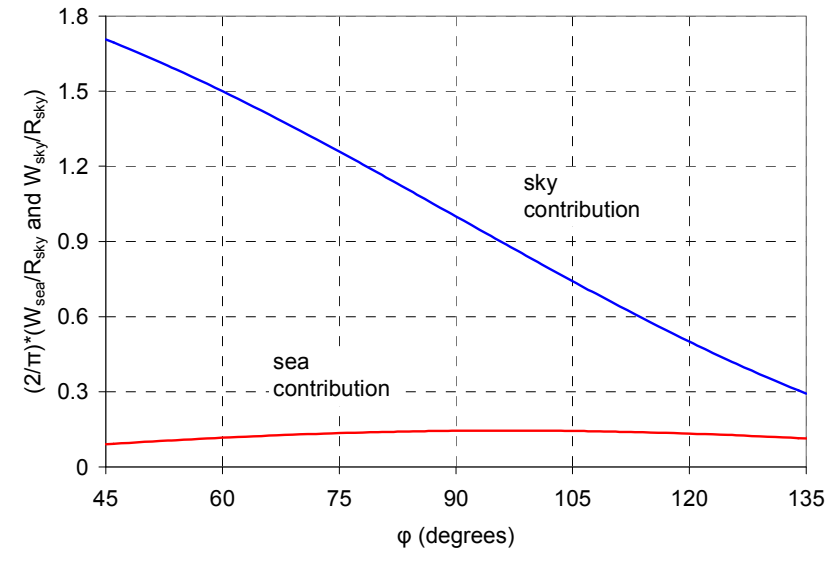

Figure 3. Comparison of object irradiances from sky and sea as function of object tilt angle $\varphi$

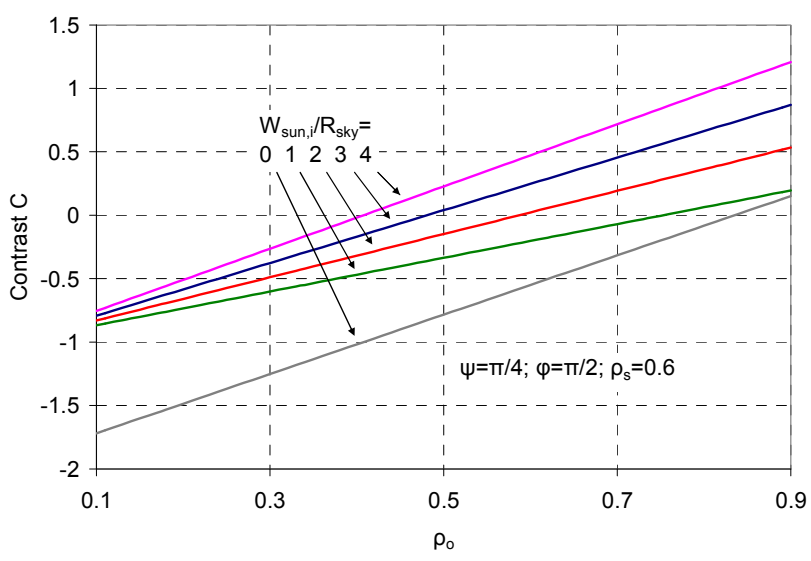

Figure 4. Contrast $\mathrm{C}$ as function of the reflection coefficient $\rho_{\mathrm{o}}$ for various $\mathrm{W}_{\text {sun }, i} / \mathrm{R}_{\text {sky }}$ values 
It is noted, that the value of $\rho_{\mathrm{s}}$ strongly depends on the sea state (or windspeed). Cox and Munk [19] give a relationship for the variance $\sigma_{\mathrm{a}}{ }^{2}$ of the wave slopes as function of the windspeed $\mathrm{w}(\mathrm{m} / \mathrm{s}): \sigma_{\mathrm{a}}{ }^{2}=0.0015+0.00256^{*} \mathrm{w}$. For a windspeed of $7 \mathrm{~m} / \mathrm{s}$ (which occurred during our experiments) we find as value for $\sigma_{\mathrm{a}}: 0.14\left(8^{\circ}\right)$, which implies an angle of incidence of $82^{\circ}$ near the horizon. There is however a lot of uncertainty, if the Cox and Munk slope distribution is valid for grazing angles, as pointed out by Mermelstein [20] and Zeisse [21]. Due to shadowing, part of the slopes will be hidden behind wave-tops. Another remark concerns the radiance of white foam at the sea surface, either produced by tumbling waves, or by the target motion. If we apply equations (1), (3) and (4) for $\varphi=0$, we find $\mathrm{W}_{\text {sky }}=\mathrm{R}_{\text {sky }} * \pi$, and by taking $\mathrm{W}_{\text {sun, },} / \mathrm{R}_{\text {sky }}=3$ and $\psi=\pi / 4$, we obtain $C=-1+1.68 *\left(\rho_{\mathrm{o}} / \rho_{\mathrm{s}}\right)$. As the reflection coefficient of the foam is always greater than 0.5 , the contrast is always positive for grazing angles. This means that target detection in the visual band is enhanced by simultaneously presence of positive and negative contrast elements. The wave-slope distribution implies also the presence of wave facets with slopes up to $0.4\left(23^{\circ}\right)$, for which the reflection coefficient reduces to about $10 \%$. This leads to the conclusion that for a moderately rough sea large variations in sea-radiance may be perceived on both sides of the average.

Other complexities arise in cases of partly clouded skies, which necessitate a more precise integration over the angles $\alpha$ and $\beta$, taking into account the sky brightness distribution. Furthermore the target consists of a number of facets under different orientation angles $\varphi$, creating target surface structure (variation in radiance) and capabilities for identification. In this case it is recommended to run a more complex model like SHIPIR [22], giving more spatial detail. This model allows also incorporation of the pixel size of the camera under consideration. The pixel size determines also the amount of averaging over the scene structure (target as well as background). It is clear, that so far we supposed, that the camera pixel was fully filled with a homogeneously distributed radiance. For longer distances this assumption does not hold, so target contrast and background clutter will both decrease beyond a certain range. Finally we can give an impression of the impact of using a polarisation filter. If we assume, that the sky radiance is not polarised (which is not quite true, see Section 6) and that the object does not polarise the sky or sun irradiance, we may conclude, that the object radiance is nearly constant, while the reflection coefficient for wave slopes of $8^{\circ}$ will fluctuate from 0.32 to 0.53 . It is thus expected, that the negative target contrast will be maximum when the direction of polarisation of the filter is horizontal, while the positive target contrast (white foam) is maximum for the vertical polarisation direction of the filter. In a similar way we may expect, outside the sun-direction, less sea-background clutter for the vertical polarisation direction.

\section{THE EXPERIMENTS}

The experiments did take place in June 2007 in the False Bay, South Africa. The observation cameras were located at the main building of IMT in Simonstown. In Figure 5, the location of Simonstown in the Bay and the observation direction is shown. It is noted that this direction is about North-East, which is in the early morning the direction of the sun. The geographic location of IMT is about $34^{\circ} 11^{\prime} 33^{\prime \prime} \mathrm{S}$ and $18^{\circ} 26^{\prime} 44^{\prime \prime} \mathrm{E}$. In the observation direction, the distance to the coast is about $20 \mathrm{~km}$. Figure 5 also shows the instrumental set-up, as positioned in a shelter on the roof of IMTat a height of about $17.5 \mathrm{~m}$ above sea level (horizon at about $14.9 \mathrm{~km}$ ). The cameras were mounted on a steerable platform.
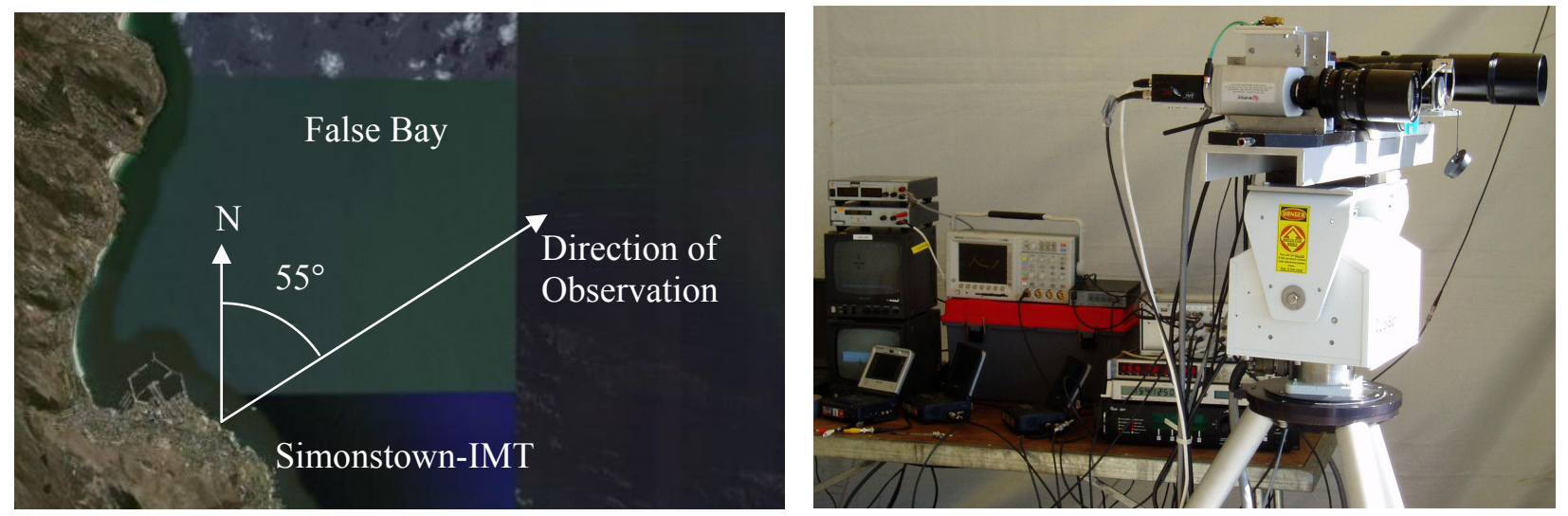

Figure 5. Left: location of the experiments in the False Bay in June 2007. Right: set-up of the TNO cameras on the roof of IMT, on a steerable tripod, close to the data recording systems 
Following is some technical data on the cameras: Imspector V9 (left); Polaroid (centre) and Marlin F-033B (right):

- Imspector; this camera, manufactured by Spectral Imaging Ltd, uses a direct-vision Prism-Grating-Prism (PGP) element with $80 \mu \mathrm{m}$ wide entrance slit (providing a spectral resolution of about $6 \mathrm{~nm}$ ), having 576 spatial points along the slit [23]. A spectral image (between 400 and $900 \mathrm{~nm}$ ) is projected on a 2/3" CCD camera with 768 horizontal pixels. The slit is vertically oriented resulting in a hyperspectral image cube by scanning the camera horizontally. It is also possible to let the target move through the projected slit. The $16-160 \mathrm{~mm}, \mathrm{f} / 2$ zoomlens was set to a focal length of $100 \mathrm{~mm}$, providing a vertical Field of View of $3.8^{\circ}$ and a pixel size of $0.12 \mathrm{mrad}$. Data recording was done on a digital DV recorder, with IRIG-B time information on the voice channel.

- Camera with rotating polarisation filter; the sensor is an I-Sight 1/2" color camera, provided with a 11.5-90 mm $\mathrm{f} / 2$ zoomlens, set to a focal length of $90 \mathrm{~mm}$. The sensor chip, with a size of $6.4 \times 4.8 \mathrm{~mm}$, provides a Field of View of $4^{\circ} \times 3^{\circ}$. The number of pixels is $768 \times 576$, which implies a pixel size of 0.09 mrad. The polarisation filter rotated with a speed of 1/7.8 revolutions per second, which means that in one revolution each polarisation direction is observed two times. The position of the filter, the time and the imagery were recorded on a digital DV recorder. It is noted, that for image analysis in Black and White, the signals of neighboring rows have to be averaged in order to remove the color "pattern-noise". The effective pixel size becomes then $0.09 \times 0.18 \mathrm{mrad}$.

- Marlin progressive scan camera; this camera has 640x480 pixels with a dimension of 10x10 $\mu \mathrm{m}$. Together with the Pentax $400 \mathrm{~mm} \mathrm{f} / 4.5$ lens, this provides a Field of View of $0.92^{\circ} \times 0.67^{\circ}$ and a pixel size of $25 \mu \mathrm{rad}$. Storage of the data, consisting of image series with 10 bits dynamic range, was done on a PC.

The target boats, that were used for data collection, discussed in this paper were the Sea Lab and Namacurra, shown in Figure 6. The Sea Lab is a two-hull laboratory boat of IMT with dimensions: 12.2x3.1 x4.0 m (Length $\mathrm{x}$ Height $\mathrm{x}$ Width) and the Namacurra is a Harbour Patrol Boat with dimensions: 10.9x1.9x2.5 m. The two boats have different paints; the paint of the Sea Lab has a higher reflection coefficient (estimated: 0.8) than that of the Namacurra (estimated: 0.4). Both paints are rather glossy, resulting in specular reflections for certain solar irradiation angles. Because of the stability of the weather conditions during the runs, we have selected the runs of 12 June (Namacurra, morning) and 13 June (Sea Lab, morning and Namacurra, afternoon). Both boats were cruising out from $1.0 \mathrm{~km}$ to $7.0 \mathrm{~km}$ and back to $1.0 \mathrm{~km}$; the Sea Lab with a speed of $3.5 \mathrm{~m} / \mathrm{s}$ and the Namacurra with a speed of $11 \mathrm{~m} / \mathrm{s}$. For the run on 12 June (around 10.30), the sun elevation was $22^{\circ}$ and the azimuth angle between the observation direction and the sun direction, was $22^{\circ}$. For the morning run on the $13^{\text {th }}$ of June (around 10.50) these values were: elevation: $23^{\circ}$; relative sun-angle: $29^{\circ}$. For the afternoon run on the $13^{\text {th }}$ (around 12.35) the values were: elevation: $32^{\circ}$; relative sun-angle: $51^{\circ}$.
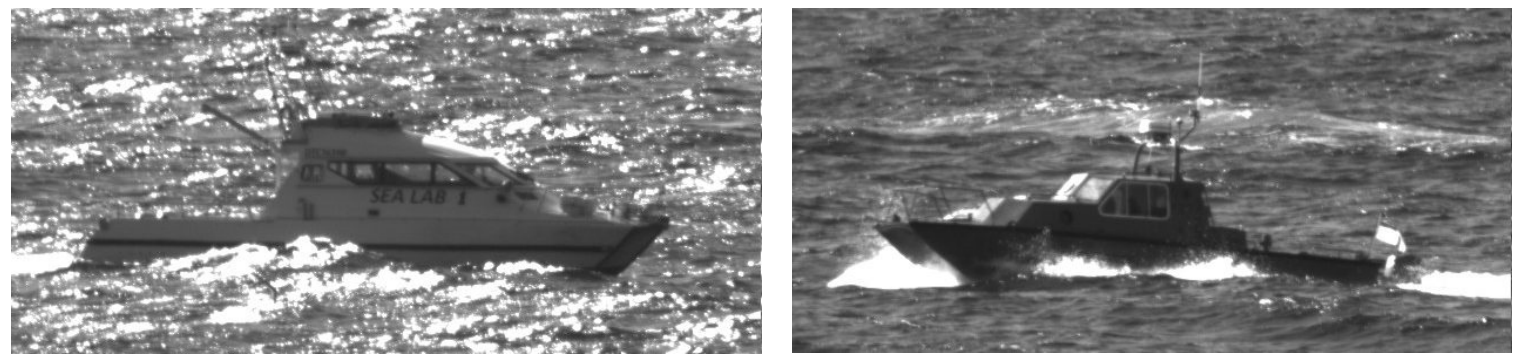

Figure 6. Close-up pictures of Sea Lab (left) and Namacurra (right), taken with Marlin camera at about $1 \mathrm{~km}$ distance

The weather and environmental conditions during the selected experiments are listed in Table 1. The air temperature and relative humidity were measured at Roman Rock, a lighthouse in the False Bay at about $2 \mathrm{~km}$ from IMT. Also the wind speed and wind direction were measured at this location. Sun and sky irradiance, including the visibility, were measured at the roof of IMT. It is noted, that the sun and sky irradiance is measured in the whole visual and near IR band, so the value is higher than the values of $\mathrm{W}_{\text {sun }}$ and $\mathrm{W}_{\text {sky }}$, introduced in the previous section and defined for the visual band. The atmospheric extinction coefficient and scintillation index are taken from the MSRT system, simultaneously measuring atmospheric propagation effects in a number of spectral bands over a $9.66 \mathrm{~km}$ range over the Bay between IMT and Muizenberg (see [15]). In this case we took the data in the spectral band around $0.6 \mu \mathrm{m}$. The blur was measured over the same range of $9.66 \mathrm{~km}$, with a high resolution camera located at IMT and a point source in Muizenberg. The blur value, given in Table 1 is specified as the Beam Wander. According to the theory of turbulence (e.g. [24]), this blur is related to the structure coefficient for refractive index $\mathrm{CN}^{2}$ via the cut-off frequency (1/e point) of the atmospheric MTF. In our case, the relation between blur and $\mathrm{CN}^{2}$ becomes: $\mathrm{CN}^{2}=\{$ blur (in $\left.\mu \mathrm{rad})\right\}^{1.67 * 1.4 \times 10^{-18}}\left(\mathrm{~m}^{-2 / 3}\right)$. 
Table 1. Weather and environmental conditions during the selected measurement periods

\begin{tabular}{|l|c|c|c|}
\hline \multicolumn{1}{|c|}{ parameter } & 12 June 10.30 & 13 June 10.50 & 13 June 12.35 \\
\hline sun + sky irradiance $\left(\mathrm{W} / \mathrm{m}^{2}\right) 0.3-2.8 \mu \mathrm{m}$ & $170-240$ & 473 & 585 \\
\hline thermal sky irradiance $\left(\mathrm{W} / \mathrm{m}^{2}\right) 5-25 \mu \mathrm{m}$ & $285-304$ & 270 & 272 \\
\hline cloud cover $($ octas) & 2 & 0 & 0 \\
\hline wind-speed $(\mathrm{m} / \mathrm{s})$ & 6 & 7 & 7 \\
\hline wind-direction $\left(\right.$ clockwise with N, in $\left.{ }^{\circ}\right)$ & 138 & 112 & 118 \\
\hline atmospheric ext. coeff. $\left(\mathrm{km}^{-1}\right) @ 0.6 \mu \mathrm{m}$ & 0.040 & 0.038 & 0.037 \\
\hline visibility $(\mathrm{km})$ & $>50$ & $>50$ & $>50$ \\
\hline blur $(\mu \mathrm{rad}) @ 9.66 \mathrm{~km}$ & 26 & 67 & 80 \\
\hline $\mathrm{CN}^{2}\left(\mathrm{~m}^{-2 / 3}\right)$ & $3.2 \times 10^{-16}$ & $1.56 \times 10^{-15}$ & $2.1 \times 10^{-15}$ \\
\hline scintillation index $=(\mathrm{std} / \mathrm{avg})^{2} @ 9,66 \mathrm{~km}$ & 0.010 & 0.093 & 0.098 \\
\hline air temperature $\left({ }^{\circ} \mathrm{C}\right)$ & 14.5 & 13.5 & 13.5 \\
\hline relative humidity $(\%)$ & 66 & 60 & 60 \\
\hline
\end{tabular}

\section{HYPERSPECTRAL DATA}

The calibration of the Imspector is somewhat problematic due to the limited dynamic range of the CCD camera ( 8 bits). This means, that on the one hand in areas of maximum response saturation may occur, while in the lower response areas (blue and near IR) the images may be noisy. In setting up the system, the first thing to do is proper alignment of the CCD camera, in order to obtain the spectrum of one point of the slit precisely on one row of camera pixels. Next the slit must be set perfectly in vertical position. Then the responsivity has to be calibrated, preferably for at least three points on the slit. In our case the responsivity versus pixel number was measured by means of a calibrated Tungsten strip lamp with a color temperature of $2600 \mathrm{~K}$ at a distance of $55 \mathrm{~m}$ in a dark tunnel. Finally the relation of pixel number with wavelength had to be calibrated, for which we used a couple of lines in the terrestrial solar spectrum: Fe/Mg at $517 \mathrm{~nm}, \mathrm{O}_{2}$ at $687 \mathrm{~nm}$ and $759 \mathrm{~nm}$ and $\mathrm{H}_{2} \mathrm{O}$ at $823 \mathrm{~nm}$. We locally used the source for the propagation experiments at a range of $9.66 \mathrm{~km}$. An example of a spectral measurement with the Imspector of this source is shown in Figure 7. The spectrum is following approximately that of a blackbody at a temperature of $3000 \mathrm{~K}$, the color temperature of the lamp. We can discriminate the two $\mathrm{O}_{2}$ and one $\mathrm{H}_{2} \mathrm{O}$ absorption lines. The increase in the spectral emission below $400 \mathrm{~nm}$ is caused by an error in the responsivity. It was found, that for this point source, the temporal signal fluctuations (scintillation) were simulataneous for all spectral points, which is not the case for all other, non-coherent scene elements in the spatial domain. This result makes the Imspector an attractive sensor for long-range multispectral transmission and scintillation measurements. It is noted, that the responsivity below $400 \mathrm{~nm}$ and above $850 \mathrm{~nm}$ is only a few percent of the maximum responsivity, which makes the accuracy of a spectral emission measurement in these areas rather low.

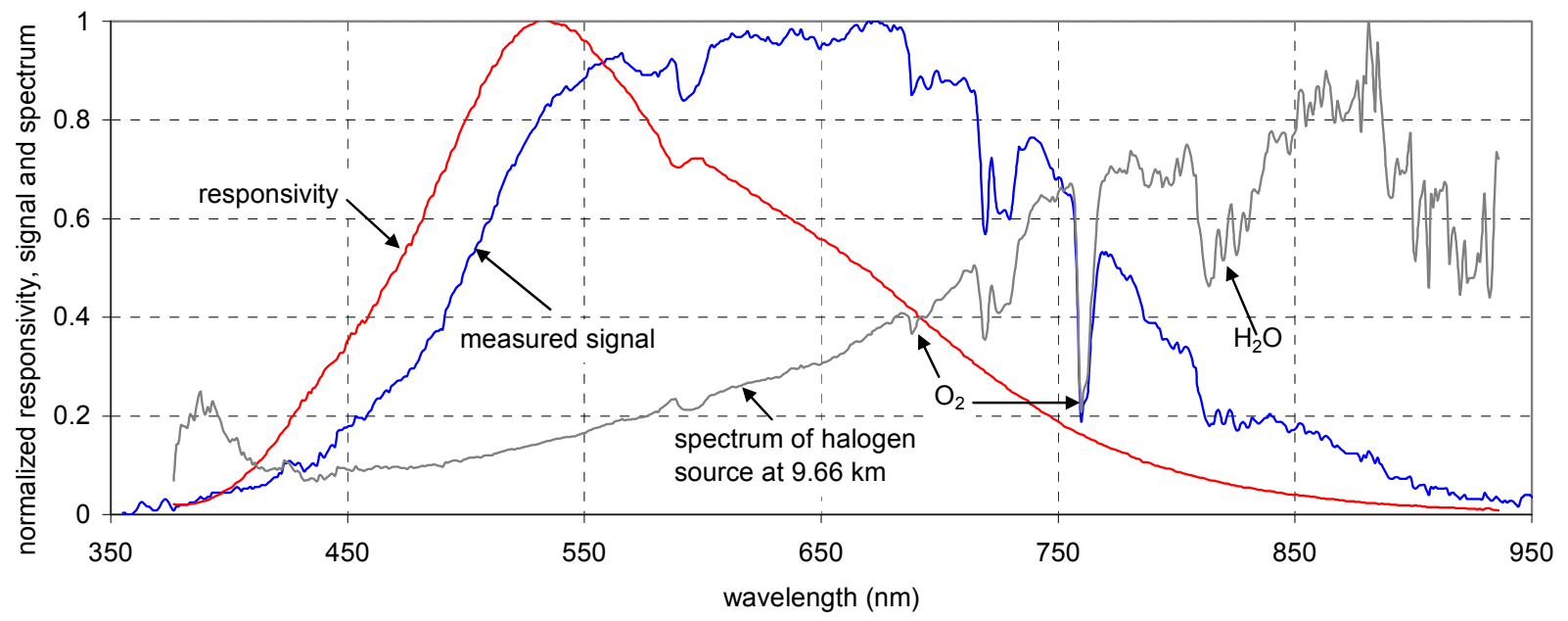

Figure 7. Spectral measurement of halogen source at $9.66 \mathrm{~km}$ range by means of Imspector on 13 June 17.22. 
Some examples of spectral emission measurements on sea foam, produced by the Namacurra at distance of $1 \mathrm{~km}$ and on sunglint are shown in Figure 8. It appears, that in both cases the relative spectral radiances are nearly 1 for wavelengths from about 420 to $830 \mathrm{~nm}$. Outside this area the uncertainty in the spectrum increases due to the lower $\mathrm{S} / \mathrm{N}$ ratio and due to the fact, that responsivity per line may differ from the averaged responsivity curve, shown in the same figure. One might expect some drop-off of the spectral radiance for wavelengths above $700 \mathrm{~nm}$, as the wavelength of the maximum solar radiance is about $500 \mathrm{~nm}$. As shown in [18] however, the solar spectral radiance appears to be rather "flat" for the whole band for air masses 3 and 4, due to the increasing atmospheric scattering at wavelengths below $1000 \mathrm{~nm}$. One may still note the two $\mathrm{O}_{2}$ absorption lines at 759 and $687 \mathrm{~nm}$.
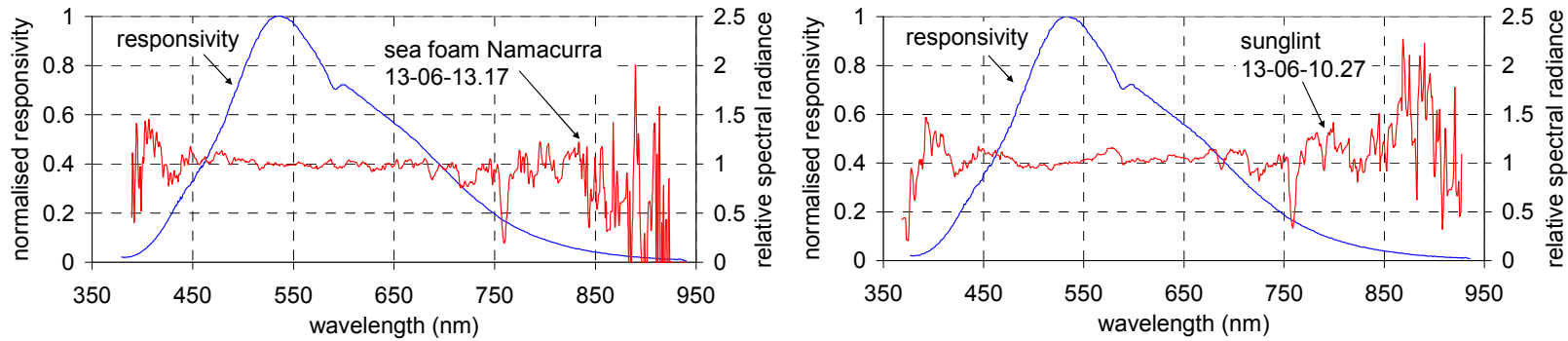

Figure 8. Samples of spectral radiance measurements on sea foam, produced by Namacurra (left), and on sunglint (right)

For a limited number of cases 3-D image cubes were produced. In the images we present a series of normal 2-D spatial pictures for about 700 wavelengths. An example 4 pictures for the wavelengths 450, 550, 650, and $750 \mathrm{~nm}$ is shown in Figure 9, where the Namacurra and its foam production can be seen. In each picture, the signals are corrected for the Imspector responsivity, while the output "gray scale" is converted to a color code: blue corresponds to low signal level and red to high signal level. In all cases the foam appears red, but the body of the boat is somewhat darker at the shorter wavelengths; it may be, that the paint reflectivity is somewhat higher above $700 \mathrm{~nm}$.
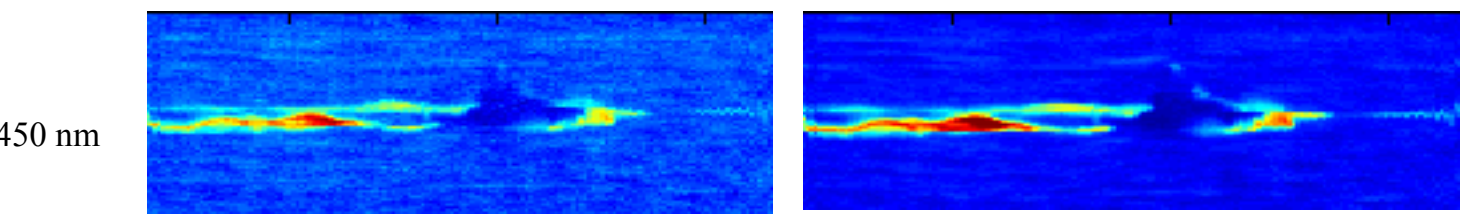

$550 \mathrm{~nm}$
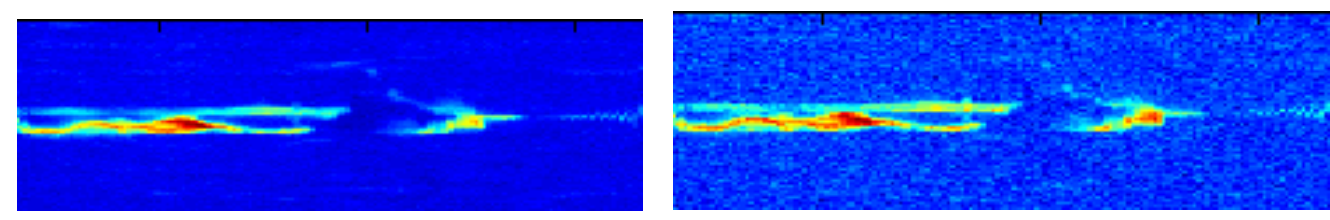

$750 \mathrm{~nm}$

$650 \mathrm{~nm}$

Figure 9. Samples from Imspector 3-D image cube: Namacurra on 13 june, 13.17; range $1.0 \mathrm{~km}$, direction $52^{\circ}$.

\section{POLARISATION-CONTRAST DATA}

Examples of images of both target boats at a range of $3 \mathrm{~km}$, are shown in Figure 10. In each set of two pictures, the picture on the left has been taken at minimum background level. The picture on the right has been taken at maximum background level. The pictures, having a size of about $15 \times 15 \mathrm{mrad}$, clearly show the different clutter conditions during the runs and also the difference between outbound and inbound situation. The presence of a negative contrast component appears in all cases, while the positive contrast component is variable due to the variations in boat motions (speed) and intervening waves. This observation is completely in line with the predictions from the model in section 2 . For each of the boat runs we have grabbed series of 150 images near each $500 \mathrm{~m}$ distance-point from 3.0 to $7.0 \mathrm{~km}$. In each series, which lasted about 6 seconds, we can observe about two images with maximum and two images with minimum intensity. This allows an analysis procedure, in which separation of contrast variations due to the rotating polarisation filter and variations due to natural effects such as foam area and target shielding by waves, can be made. 

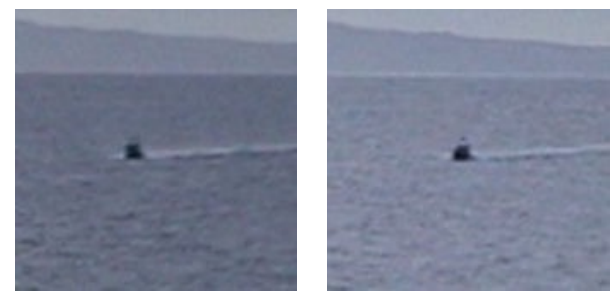

12 June 10.40.51, Namacurra inbound
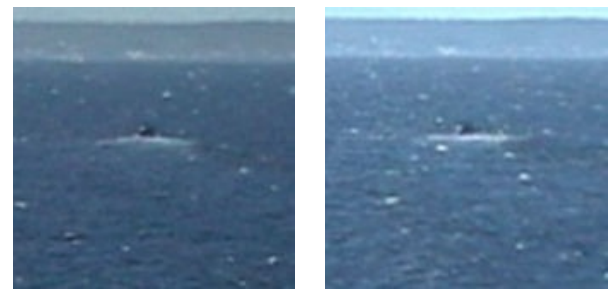

13 June 12.20.15, Namacurra outbound
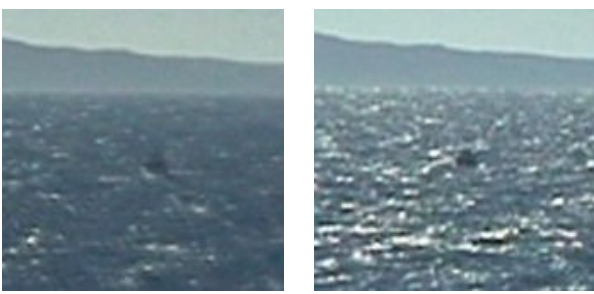

13 June 10.32.16, Sea Lab outbound

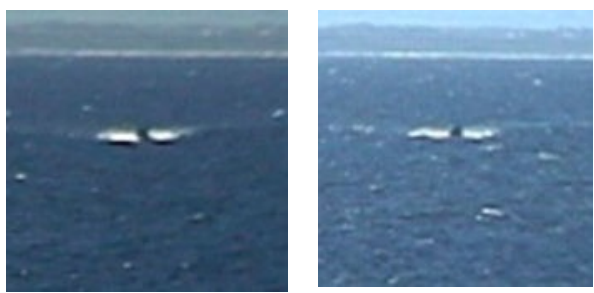

13 June 12.44.45, Namacurra inbound

Figure 10. Image samples, taken with CCD camera + polarisation filter, of inbound and outbound target boats at $3 \mathrm{~km}$

During the image analysis, we determined for an area around the target and a neighboring reference area, the Difference of Summed signal levels of enclosed pixels (DOS), the minimum and maximum signal level in the target area (FgMin and FgMax) and the mean background level (BgAvg). The DOS value represents the sum of the bit values for all pixels in the target area minus the sum of the bit values of the same number of pixels with the BgAvg level. This sum can be positive and negative; a zero value may however not lead to the conclusion, that the target is invisible, as positive and negative contrast elements may cancel each other. In Figure 11 three representative plots of the four parameters versus frame number are shown. Strong fluctuations in the DOS level is observed, caused by the effects, mentioned before. The FgMin level is more constant and apparently not much modified by the polarisation filter. Some variation may occur at longer ranges due to the fact, that the dark area of the target becomes smaller than a pixel. Similar variations occur in the FgMax level in case of small whitecap areas. The BgAvg level shows a nice sinusoidal variation with time, as could be expected. It was found however, that the minimum signal level did not correspond with a vertical position of polarisation direction of the filter, especially for the images of 13 June (see also next section).
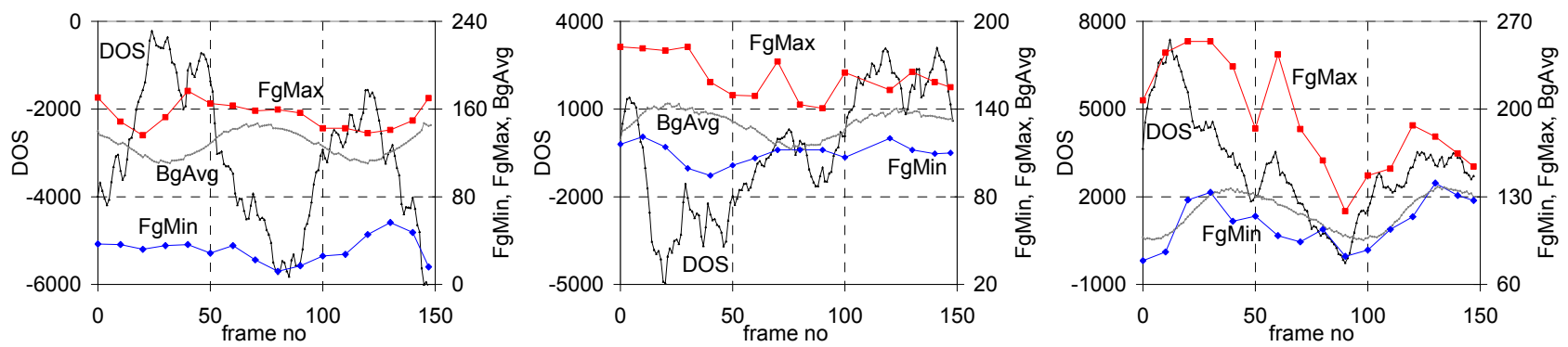

Figure 11. Sample data on DOS, FgMin, FgMax and BgAvg as function of frame number (time) for different inbound runs on 12-06-10.40 $(3.5 \mathrm{~km}), 13-06-10.56(6.5 \mathrm{~km})$ and 13-06-12.39 $(6.5 \mathrm{~km})$

It was also found that the sky radiance was polarised, as may be expected for directions out of the sun direction. Finally we noticed a second harmonic in the sinusoidal behaviour of BgAvg on the $13^{\text {th }}$, probably due to the fact, that for the given wind direction $\left(115^{\circ}\right)$, the normals of the wave facets were tilted from vertical plane through the observation direction $\left(55^{\circ}\right)$. From the selected image series, contrast data were determined in terms of maximum and minimum DOS values for each series as well as maximum differences of the negative and positive contrast components in the target area, compared to the BgAvg level. The target area did vary somewhat, but was in general about $6 \times 3 \mathrm{mrad}$, not too large, in order to exclude spurious clutter elements, but large enough to contain most of the foam produced by the target. 
In Figure 12 contrast plots of the DOS (maximum and minimum value) and maximum positive/negative signal difference with the BgAvg level in each series of 150 frames, are presented for the three selected boat runs, outbound and inbound, for ranges between 3 and $7 \mathrm{~km}$. It is shown, that at the maximum range of $7 \mathrm{~km}$, the DOS contrast is decreasing to values close to zero. Considering the fluctuations in the DOS level due to background clutter, we conclude that the detection range for this type of camera, based upon the DOS criterion, is close to $7 \mathrm{~km}$. This range compares well with a rule of thumb for the range where the pixel size (including camera blur) equals the boat size in front aspect. The detection range for inbound and outbound runs is about the same. Observation of the lower plots in Figure 12 tells us, that the contrasts of the peak signal (positive as well as negative) allow a longer detection range. It is noted, that in most cases the negative target signal is giving the highest contrast at maximum BgAvg level. Similarly the positive target signal is giving the highest contrast at minimum BgAvg level. From this we may conclude, that the use of a polarisation filter makes sense.
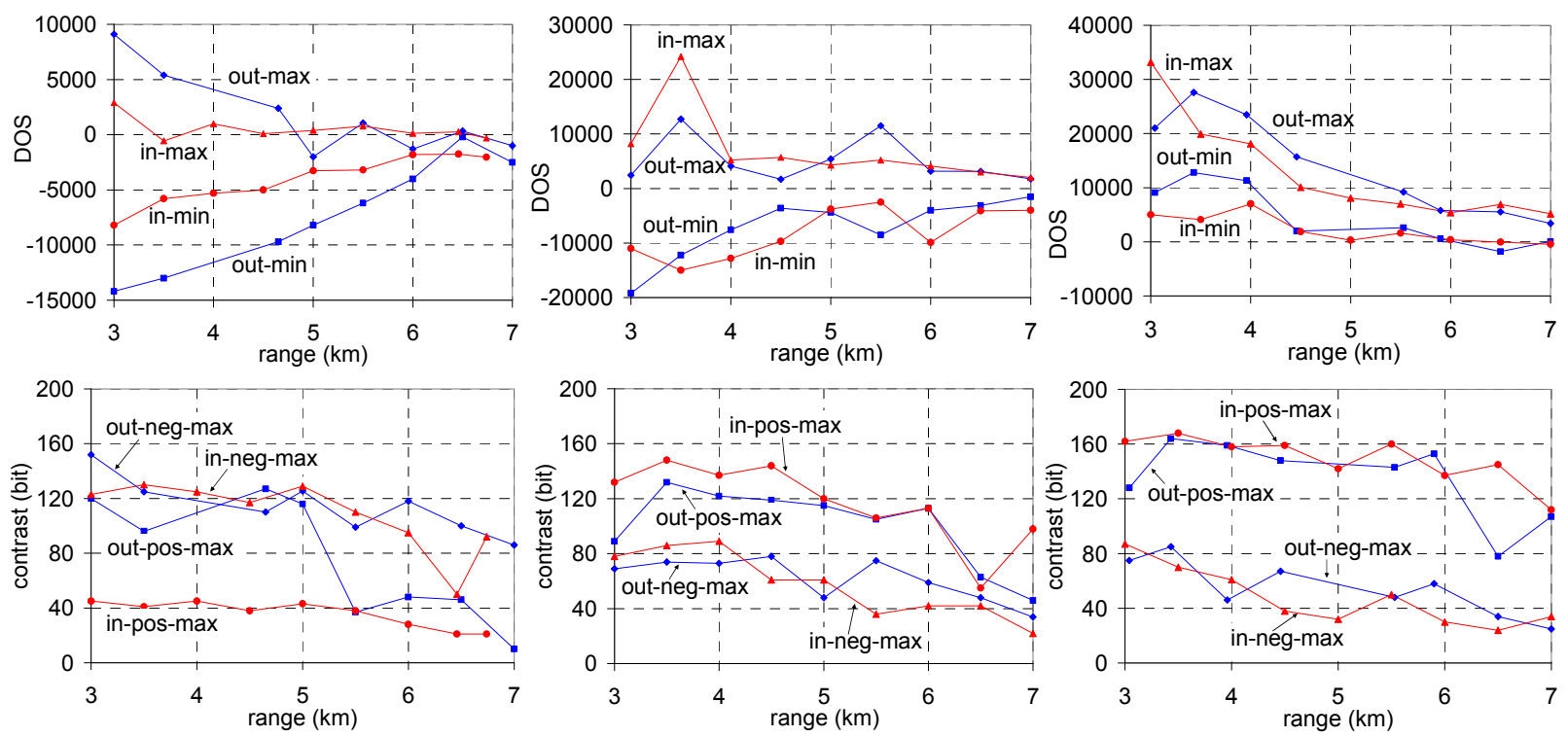

Figure 12. Contrast data for the three boat-runs on 12 June (left), 13 June (morning, centre), 13 June (afternoon, right)

The rotation of the polarisation filter gives an additional key for target detection, complementary to the natural contrast variations, caused by the variable target cross section and variations in the amount of sea spray, produced by the target in motion. It is obvious, that the white foam trail, observable behind the target in calm sea conditions such as in the case of the incoming Namacurra on the $12^{\text {th }}$, gives some stability in the contrast, especially if the target is not precisely head on. It was found, that at different places in the Field of View, the position of the filter for maximum- and minimum signal level was not everywhere the same. This was apparently caused by minor variations in the surface structure of the sea over the Field of View, probably correlated with local variations in wind patterns. An interesting issue is the existence of a special signature of the incoming target at high speed, composed of a dark central area with two white foam areas on the left and on the right, caused by the bow waves, as can be seen in Figure 10 for the $13^{\text {th }}$ for the Namacurra.

\section{POLARISATION-CLUTTER DATA}

In an earlier paper ([14]), infrared background clutter data were presented for the same experiments, to be used as inputs for the MIBS background simulator. In this section we will present the spatial variation of visual background clutter with elevation (down-look angle in the image below the horizon). We have special interest for longer ranges, where clutter elements become smaller than the camera pixel. This, together with the obscuration effect of waves, leads to less clutter, as was mentioned before in section 2. Next, the effect of the polarisation filter on the clutter magnitude will be illustrated and finally clutter histograms will be shown for the imagery taken during the selected runs. These histograms play a key role in the prediction of the probability of false alarms (false detections). Again we are trying to find out if the benefit of using a polarisation filter, (improved target detection capability) in terms of clutter reduction. 

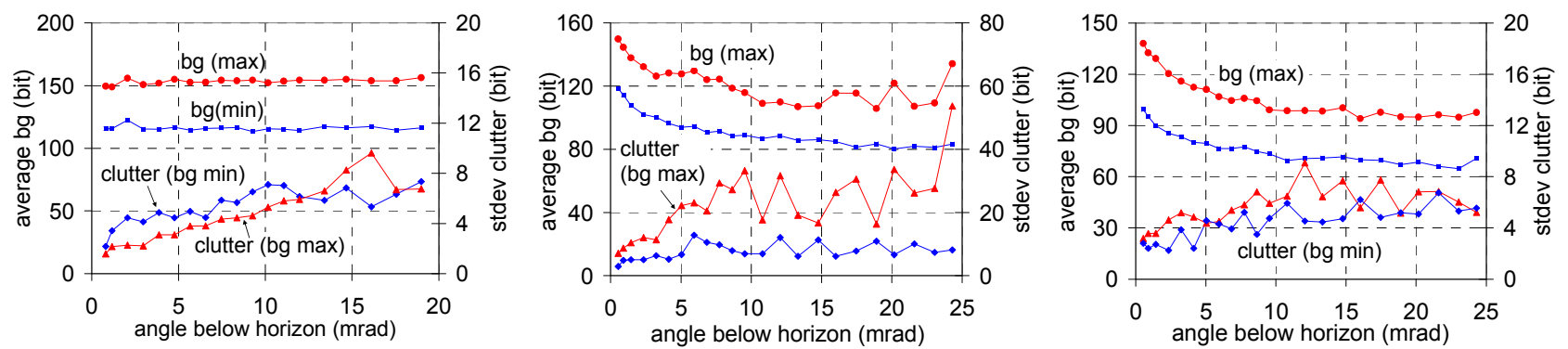

Figure 13. Average background level and background clutter as function of elevation for data of $12 \mathrm{June}(10.28$, left), 13 June (10.48, centre) and 13 June (12.39, right) for two polariser positions (minimum and maximum BgAvg)

In Figure 13 background and clutter data are presented as function of elevation (angle below horizon) for three typical images, taken during the selected runs. The data concern the average and standard deviation of a row of pixels in an area of $0.18 \times 27 \mathrm{mrad}$ at a number of elevations for two positions of the rotating polarisation filter (maximum and minimum background level). First of all the spatial noise of the camera for the sky background was determined. For the three runs we found a mean value of 1.32 bits, far below the sea clutter. The clutter behaviour is very different for the three runs, as might be expected from the differences in weather- and environmental conditions, listed in Table 1. For the run on the $12^{\text {th }}$, the clutter level increases for larger angles below the horizon, while the background level stays about the same. The clutter level is about the same for maximum and minimum background conditions. This is not the case for the morning run on the $13^{\text {th }}$, when the camera was looking more into the sun direction. Here the clutter level is less for the minimum background condition. We find also a decrease of the average background level for increasing angles below the horizon. This is also the case for afternoon run on the $13^{\text {th }}$, where we also find similar clutter levels for the two background cases.
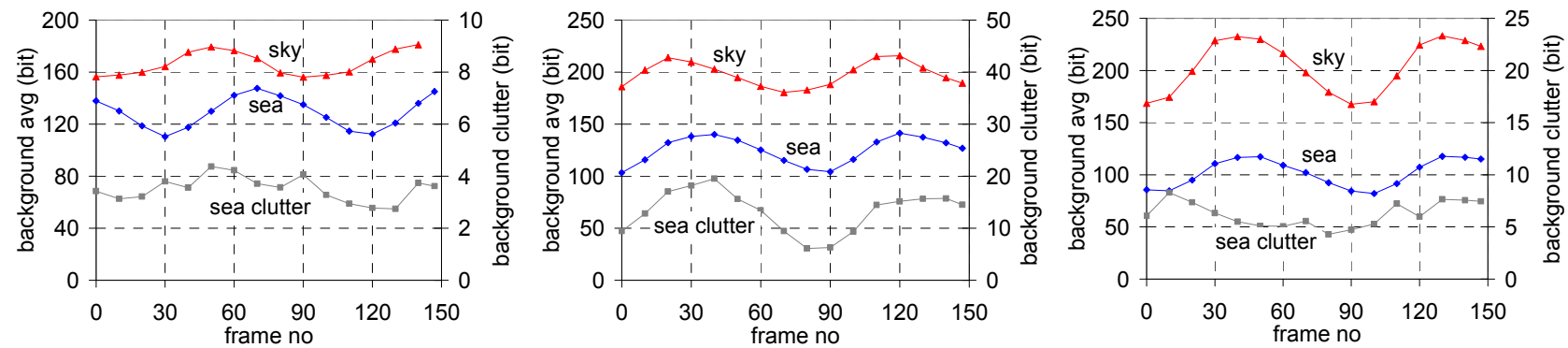

Figure 14. Background clutter and sea and sky background level as function of frame number for 12 June (10.40, left), 13 June (11.10, centre) and 13 June (12.43, right); data taken in FOV: 0.9x18 mrad, at target elevation.

Next, the background radiance and the background clutter level is considered. Some representative data are shown in Figure 14. Similar to Figure 11, we have put on the horizontal axis the frame number, being equivalent to time, as 150 frames are passing in about 6 seconds. It is noted, that the start of the series of frames is arbitrarily, so for each series, the position of the polarisation filter is vertical for a different frame number. These frame numbers were determined from the time-trigger pulses on the voice channel of the DV recorder. For the interest of the reader we have included in Figure 14 the variation of the sky background. Comparison with the sea background in the same series of frames tells us, that both plots are shifted a number of frames; the shift is not the same for the three different runs. This means that the orientation of the polarisation of the sky radiance is different from that of the sea radiance.

The sea background radiance and clutter level were measured at the same elevation as the target, which was incoming at a range of $3.5 \mathrm{~km}$, which implies a down-look angle below the horizon of about $3.2 \mathrm{mrad}$. The size of the "window", in which the data were analysed, was $0.9 \times 18 \mathrm{mrad}$, while the location was on the left side of the target. The behaviour of the clutter as function of frame number is apparently different for the three occasions. In "out of the sun" directions, as found in the left and right plots, we observe some clutter variations, mainly due to variations in the wave structure and white cap concentrations in the relatively small window. A strong correlation of the clutter and background variation is observed in the centre plot. For this run the relative sun-angle was $23^{\circ}$, implying sunglints, as shown in Figure 10. 
Apparently the polarisation filter considerably reduces the clutter level in directions about $30^{\circ}$ left and right of the sun direction. Light, reflected by the wave facets of the sea surface in this area, is highly polarised in roughly horizontal directions. It is noted here, that according to Cox and Munk [19], the normal vectors to the wave facets are randomly oriented in two directions with different standard deviations in wind- and crosswind directions. The wave facets, being present in one pixel area, have a mean orientation, which may vary from one pixel to the neighboring pixels and thus produce minimum reflections in different pixels and different frames due to the time dependence of the phenomenon. Figure 15 shows a different way of quantifying clutter via the histogram analysis. The plots for the $12^{\text {th }}$ (left) show very similar distributions, with standard deviations of 3.7 and 4.0 bits for the minimum respectively maximum background average. For the data of the morning run on the $13^{\text {th }}$ (centre) we find a major difference due to the sunglint effect, with standard deviations of 6.1 respectively 19.7 bits. It is noted however, that for the frame of maximum background average pixels are found with high signal levels. This means, that the probability of false alarm is quite high for this frame, while this is much less for the frame with minimum background level. Even for the afternoon frame on the $13^{\text {th }}$ (right) we find this kind of situation, although the standard deviations are less: 5.3 respectively 8.8 bits.
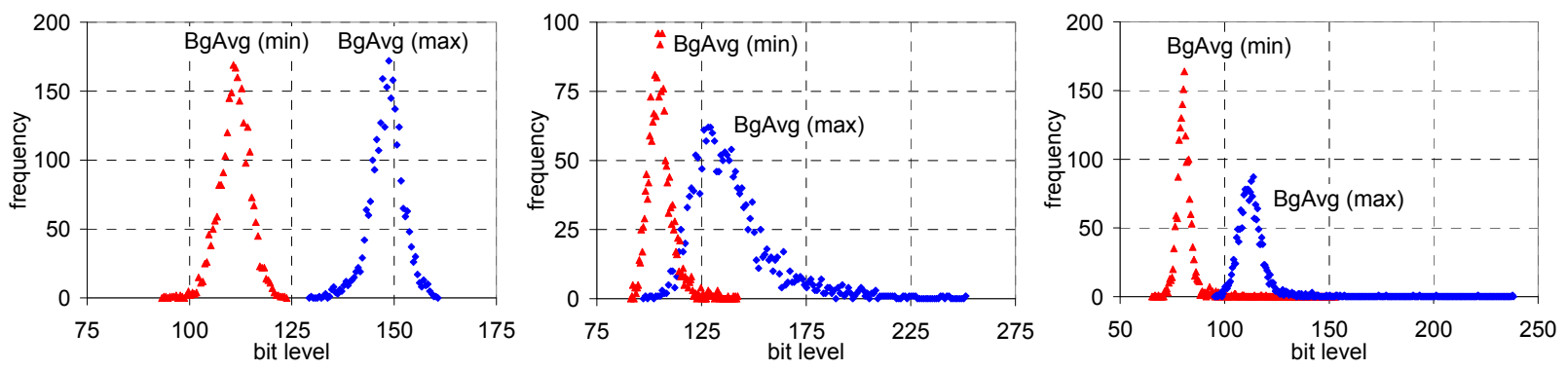

Figure 15. Histograms of background clutter for the frames of maximum and minimum background average for the same series of images as taken in Figure 14. The window size of the clutter data was $2 \times 18$ mrad.

Next we will consider the question about the benefit of using a rotating polarisation filter. A summary of the effects of the filter on the target contrast and background average and -clutter is given in Table 2. The polarisation angle $\theta$, defined as the clockwise-measured angle between the direction of polarisation of the filter and the vertical, the Polarisation Index PI (see also Figure 1) and the peak-peak DOS clutter, as measured in two neighbouring sea backgrounds (window size about $2.5 \times 2.5 \mathrm{mrad}$ ), are presented for the three selected runs. The data for $\theta$ and PI are mean values for all outbound and inbound ranges, while the standard deviation is given as well. It is noted, that the sky radiance is not vertically polarised. This means, that in addition to the deviation of the orientation of the normals of the wave facets from the vertical, the sky also has an impact on the values of $\theta$ for the maximum and minimum sea background level. We find also, that for the three runs, the values of $\theta$ are significantly different, but rather consistent for all ranges of one run. This means, that the use of a polarisation filter is beneficial for optical target detection, and that with the support of suitable background knowledge, the contrast as well as the value of $\theta$ for optimum contrast is predictable. Of course we have to remind, that the contrast modulations, produced by the filter, are mixed with random contrast variations by the background.

Table 2. Summary of measured data on the polarisation angle $\theta$, Polarisation Index PI and background DOS clutter

\begin{tabular}{|l|c|c|c|}
\hline \multicolumn{1}{|c|}{ parameter } & 12 June morning & 13 June morning & 13 June afternoon \\
\hline$\theta$ for max. sea Bg. level & $55^{\circ}+/-12^{\circ}$ & $40^{\circ}+/-12^{\circ}$ & $18^{\circ}+/-4^{\circ}$ \\
\hline$\theta$ for min. sea Bg. level & $-20^{\circ}+/-19^{\circ}$ & $-38^{\circ}+/-8^{\circ}$ & $-52^{\circ}+/-3^{\circ}$ \\
\hline$\theta$ for max. neg. contrast & $77^{\circ}+/-17^{\circ}$ & $52^{\circ}+/-20^{\circ}$ & $99^{\circ}+/-45^{\circ}$ \\
\hline$\theta$ for max. pos. contrast & $-19^{\circ}+/-33^{\circ}$ & $-54^{\circ}+/-38^{\circ}$ & $-48^{\circ}+/-34^{\circ}$ \\
\hline$\theta$ for max. sky Bg. level & $20^{\circ}$ & $19^{\circ}$ & $9^{\circ}$ \\
\hline$\theta$ for min. sky Bg. level & $104^{\circ}$ & $121^{\circ}$ & $111^{\circ}$ \\
\hline PI for max. and min. Bg. level & $0.25+/-0.01$ & $0.27+/-0.02$ & $0.34+/-0.02$ \\
\hline PI for neg. contrast pulse & $0.53+/-0.13$ & $0.35+/-0.13$ & $0.36+/-0.16$ \\
\hline PI for pos. contrast pulse & $0.24+/-0.13$ & $0.48+/-0.17$ & $0.59+/-0.26$ \\
\hline pp Bg. DOS clutter 3 km & 3300 & 11350 & 3050 \\
\hline pp Bg. DOS clutter 6 km & 990 & 3500 & 1350 \\
\hline
\end{tabular}


The PI values for the background radiation, as shown in Table 2, correspond approximately with the values in the PI plot in Figure 1 for angles of incidence of about $85^{\circ}$. This is an indication for the average tilt angle of the wave facets within one pixel of about $5^{\circ}$. The PI values of the positive and negative contrast pulses are reasonably high, confirming the idea about the benefit of using a polarisation filter. It is noted, that the PI values for the contrast pulses are different for the three runs due to the differences in weather conditions. The DOS clutter decreases rapidly with range due to increased averaging of the clutter elements over the pixel area for longer ranges. Comparing the peak-peak clutter values from Table 2 with the DOS contrast plots in Figure 12 shows, that even detection of small targets by using the DOS criterion, provides sufficient signal to clutter ratio up to ranges of $7 \mathrm{~km}$.

\section{TARGET IDENTIFICATION}

In this section we will show some results of the identification experiments, carried out with the Marlin camera. It is also investigated, if the identification is influenced by the conditions of atmospheric turbulence. These conditions were quite different on the $12^{\text {th }}$ and $13^{\text {th }}$ of June (measured blur spots of 26 resp. $80 \mu \mathrm{rad}$ ). For this purpose, the target boats were making circle runs at ranges of 1,3,5 and $7 \mathrm{~km}$. For these runs we took series of movies, from which good stills were selected (avoiding images with too much sea spray over the target). We selected the Namacurra images of the two days and reduced the picture size for the $1 \mathrm{~km}$ range to $5.25 \times 5.25 \mathrm{mrad}$. For the other ranges we zoomed in 3,5 and 7 times, in order to keep the target size the same. The result of this exercise is shown in Figure 16. In the (nose on) images, taken at a range of $1 \mathrm{~km}$, we observe many spatial details, disappearing at longer ranges due to the decrease in the number of pixels on the target, the MTF (Modulation Transfer Function) of the camera (including vibrations and integration time), target motions and due to the atmospheric blurring. One significant spatial detail is the curved fixture on top, carrying the communication and navigation box. On the images of the $12^{\text {th }}$, this construction is observable at ranges of $7 \mathrm{~km}$. For the imagery on the $13^{\text {th }}$, this item is lost beyond a range of $3 \mathrm{~km}$. At the range of $7 \mathrm{~km}$ on the $13^{\text {th }}$, the target is observable as one "blob". This result confirms the expectations from the blur measurements with the Celestron camera ([15]).
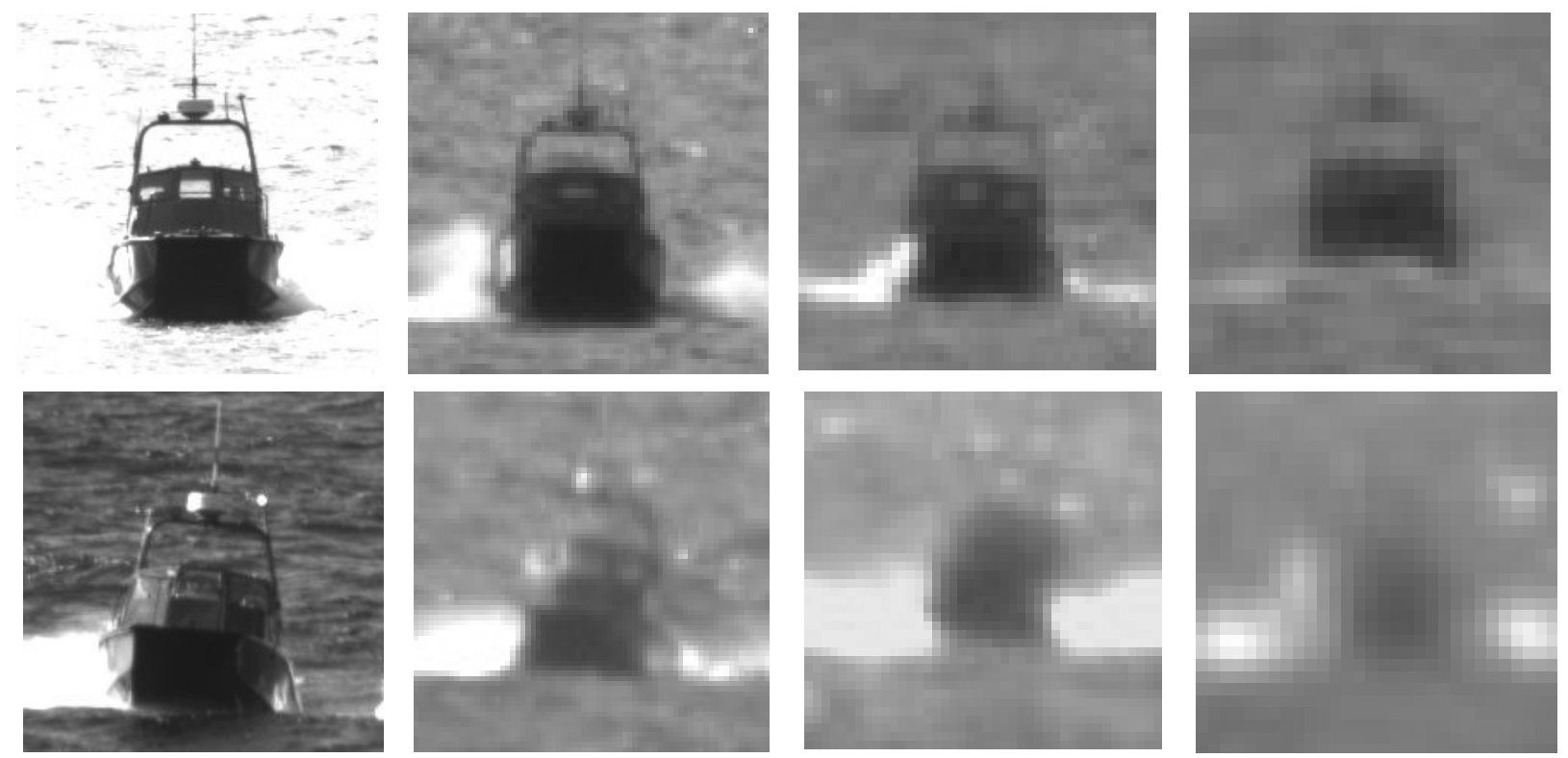

Figure 16. Imagery, taken with the Marlin camera, of the Namacurra (head on) at ranges of 1,3,5 and $7 \mathrm{~km}$ (from left to right) during the runs of 12 June (top, low turbulence) and 13 June (bottom, high turbulence)

Without atmospheric blur, the identification range, given the target size $(2.5 \times 2.5 \mathrm{~m})$ and the resolution of the camera (estimated effective spot size, including the lens: $35 \mu \mathrm{rad}$ ), can be determined, following the guide lines on the concept of Minimum Resolvable Contrast (MRC), described by Holst [25]. For identification, we need about 14x14 effective pixels on the target (7 line-pairs, according to the MRC), which occurs at a range of $5.1 \mathrm{~km}$, corresponding reasonably well with the details, observed in the image of the $12^{\text {th }}$. 
If we take into account the atmospheric blur, we realise that the blur $\mathrm{B}_{\mathrm{R}}$ at range $\mathrm{R}(\mathrm{km})$ is related to the blur $\mathrm{B}_{9.7}$ at the range of $9.7 \mathrm{~km}$ (Table 1 ) by: $\mathrm{B}_{\mathrm{R}}=\mathrm{B}_{9.7} *(\mathrm{R} / 9.7)^{0.6}$. At a range of $5 \mathrm{~km}$ on the $12^{\text {th }}$ and $13^{\text {th }}$, the blur is thus 17 respectively $54 \mu \mathrm{rad}$. It is clear, that the latter value does not allow identification at a range $5 \mathrm{~km}$, which we can verify in Figure 16. Another example of imagery of the Namacurra (port side view) at a range of $5 \mathrm{~km}$ on both days, leading to similar conclusions, is shown in Figure 17. Thanks to the larger projected area, the target can just be identified on the $13^{\text {th }}$.
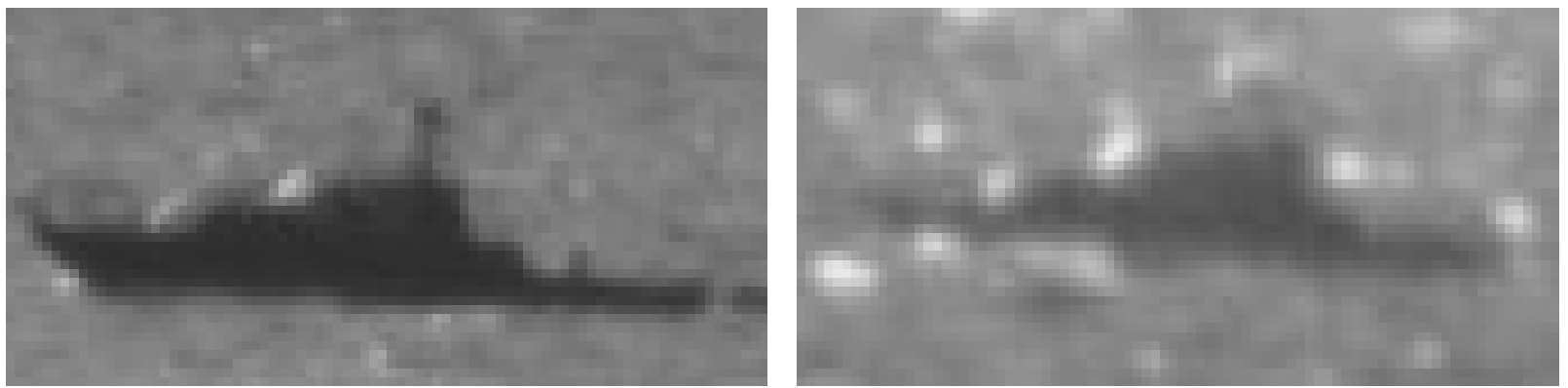

Figure 17. Comparison of Marlin pictures of the Namacurra (port side) at $5 \mathrm{~km}$ on 12 June (left) and 13 June (right)

\section{CONCLUSIONS}

In this paper we have presented a simple model to predict the visual contrast of small targets at sea, using basic weather input data. Results are presented of images, collected with three different types of cameras: one hyperspectral camera, one camera with a rotating polarisation filter in front of the lens and one high resolution for target identification. Images were analysed from three selected boat runs on two days with different environmental (irradiance) conditions. From the data, the following conclusions are drawn:

- The simple contrast model appears to be a useful tool for understanding the observed target contrast effects

- The hyperspectral data show a nearly perfect "white" spectrum for the background clutter elements sun-glints and white caps. Therefore this type of sensor may help to reduce the false alarm rate, if adequately applied

- In the imagery from the polarisation camera, we observed two types of contrast modulation: one natural, caused by the variability of the spray, produced by the motion of the target and one, resulting from the rotation of the polarisation filter.

- The target always shows a positive and a negative contrast element in contrary to the imagery from thermal IR sensors, for which the contrast is nearly always positive. It has been shown, that the polarisation filter produces a maximum contrast for both contrast elements.

- It was found, that the polarisation angle of the filter for maximum contrast and for maximum and minimum background level was consistent for each run

- The use of a rotating polarisation filter in front of visual cameras for detection of small surface targets at sea is therefore recommended. The speed of the filter should be between 5 and 10 seconds per revolution

- The sea-clutter may be reduced at certain filter positions, especially for perception near the direction of the sun, in the presence of dominant sun-glints. In most other conditions the sea-clutter is less modulated by the filter

- The sky radiance was also polarised during the experiments, where the polarisation angle for minimum sky radiance was found to be systematically different from the polarisation angle for minimum sea radiance. This latter angle was significantly different from the expected angle of zero degrees. This is probably caused by the deviation of the tilt angle of the wave facets from the vertical.

- Significant differences in the histograms of the sea-clutter were found for the three selected runs. Also the histograms for maximum and minimum average background radiation were quite different. This result has an impact on the false alarm rate during the detection process for long-range small surface targets.

- The use a detection criterion with positive and negative thresholds is preferred to the DOS criterion for target detection. The latter may produce occasionally zero total contrast, if the sum of the positive contrast elements is the same as that from the negative elements. Combination of both detection criteria is however recommended

- The imagery, taken for target identification, showed a major blurring effect during one of the days, caused by atmospheric turbulence; this was in agreement with quantitative blur measurents, carried out simultaneous. 


\section{ACKNOWLEDGEMENTS}

The work for this paper was supported by the Netherlands MOD under the programme V602 "Electro-Optical sensor systems". The South African Navy is greatly acknowledged for providing the target boats and performing the proposed runs. The personnel of IMT is acknowledged for the general assistence during the trials period. We thank our colleague Herman Bekman at TNO, for preparing software for the analysis of the hyperspectral data.

\section{REFERENCES}

[1] Arie N. de Jong et al, Small surface target detection with EO/IR sensors, Proceedings $3^{\text {rd }}$ NATO-IRIS conference, Quebec, October 1998

[2] Arie N. de Jong, Results of Nettuno maritime IR experiments; July 1998, TNO report FEL-99-A086, February 2000

[3] Arie N. de Jong et al, Report on the Baltic 99 experiments, TNO report FEL-00-A094, September 2000

[4] Piet B.W. Schwering et al, Results of the Warm-humid Thermal Imager Experiment WATIX, TNO report FEL-00A116, October 2000

[5] Sebastiaan P. van den Broek et al, Detection and classification of infrared decoys and small targets in a sea background, SPIE Vol. 4029, Targets and Backgrounds VI, Orlando, April 2000

[6] Arthur D. van Rheenen et al, Measurements of the vertical radiance profile using infrared sensors, SPIE Vol. 6747, Optics in Atmospheric Propagation and Adaptive Systems, Florence, September 2007

[7] Piet B.W. Schwering, IRST evaluation methodologies: Maritime Infrared Background Simulator MIBS, SPIE Vol. 6206, Infrared Technology and Applications XXXII, Orlando, April 2006

[8] Arie N. de Jong et al, IR detection of very small invading surface and sub-surface targets in littoral waters, Proceedings $5^{\text {th }}$ MSS symposium, Gaithersburg, December 2002

[9] Arie N. de Jong, IR Threat Detection Systems (IRTDS), SPIE Short Course SC 628, Orlando, April 2006

[10] Sebastian P. van den Broek et al, Discriminating small extended targets at sea from clutter and other classes of boats in infrared and visual light imagery, SPIE Vol. 6969, Orlando, March 2008

[11] Henri Bouma et al, Automatic detection of small surface targets with Electro-Optical sensors in a harbour environment, SPIE Vol. 7114, Cardiff, September 2008

[12] Arie N. de Jong, Multispectral maritime background- and clutter effects on small surface target detection, SPIE Vol. 2742 Orlando, April 1996

[13] Piet B.W. Schwering et al, Optical Characterisation of small surface targets, SPIE Vol. 6739A, Florence, September 2007

[14] Piet B.W. Schwering et al, IRST infrared background analysis of bay environments, SPIE Vol. 6934, Infrared Technology and Applications XXXIV, Orlando, March 2008

[15] Arie N. de Jong et al, Measurement of optical refraction-, transmission- and turbulence effects in the False Bay, South Africa; June 2007, SPIE Vol. 7108, Optics in Atmospheric Propagation XI, Cardiff, September 2008

[16] F. Cremer et al, Infrared polarisation measurements of targets and backgrounds in a marine environment, SPIE Vol. 4370, Targets and Backgrounds VII: Characterisation and Representation, Orlando, April 2001

[17] Max Born and Emil Wolf, Principles of Optics, $7^{\text {th }}$ edition (page 44), Cambridge University Press, January 1999

[18] David Kryskowski et al, Sources of Radiation: Natural Sources (pages 151-162), The Infrared \& Electro-Optical Systems Handbook, Volume 1, SPIE Optical Engineering Press, Bellingham, 1996

[19] Charles Cox et al, Measurement of the Roughness of the Sea Surface from Photographs of the Sun's Glitter, JOSA Volume 44, Number 11, November 1954

[20] M.D. Mermelstein et al, Infrared radiance and solar glint at the ocean-sky horizon, Applied Optics, Vol. 33, No. 25 September 1994

[21] Carl R. Zeisse, Radiance of the ocean horizon, JOSA A, Vol. 12, No. 9, September 1995

[22] David Vaitekunas, ShipIR/NTCS Training Module, Davis Engeneering Limited, Version 2.7, Ottawa, June 2000

[23] Spectral Imaging Ltd. User Manual of Imspector, Version 2.21 (August 2003)

[24] Robert R. Beland, Propagation through Atmospheric Optical Turbulence (page 191), The Infrared \& ElectroOptical Systems Handbook, Volume 2, SPIE Optical Engineering Press, Bellingham, 1996

[25] Gerald C. Holst, Electro-Optical Imaging System Performance, $3^{\text {rd }}$ ed. SPIE PM 121, Bellingham, 2003 